\title{
Conceptualization of the photon for quanta of structured light
}

\author{
David L. Andrews \\ University of East Anglia, Norwich NR4 7TJ, United Kingdom
}

\begin{abstract}
The development of optical modes with intrinsic structure has significantly redirected attention to the established concept and fundamental physics of the photon. In particular, to accommodate the modal structure of beams with transverse structure, two aspects of conventional representation invite special modification: the mathematical formulations, and figurative depictions. Reappraisals of the former highlight known deficiencies in simple plane wave mode expansions, at the price of associating the properties of individual quanta with macroscopic beam parameters wrought by the physical optics. Experimental proofs that such features do indeed reside in individual photons lead to conceptual problems, yet it is clear that alternative viewpoints engender still deeper difficulties. Such quandaries are also evident in the variety of diagrammatic representations used for structured radiation. Though such depictions cannot ever adequately represent the quantum physics, their incautious use can become seriously misleading, misrepresenting the underlying mathematics and supporting wrong conclusions. This analysis aims to expose and underscore some issues that invite closer attention.
\end{abstract}

Keywords: Structured light, complex light, twisted light, optical vortex, photonics, photon interactions, quantum optics, optical modes, quantum communication, localization, spatial light modulators

\section{INTRODUCTION}

It is clearly evident, from the technical development history of optical modes with intrinsic wavefront and polarization structure, that the accompanying and ensuing renaissance in laser optics has led to a remarkably broad reappraisal of the established concept and physics of the photon. ${ }^{1-4}$ In order to accommodate forms of transverse beam structure, two aspects of conventional representations in optics have invited special modification: the mathematical formulations, and figurative depictions. Whilst a common focus of attention on the former quickly highlights well-known deficiencies and limitations in the physical realism of simple plane wave mode expansions, resolution of these problems immediately raise a new, specific difficulty in the quantum formulation: individual photons must represent quanta of modes that are defined by macroscopic beam parameters, determined by the physical optics. As envisaged in original work on vortex beams, ${ }^{5}$ it now appears incontrovertible that such features do indeed reside in individual photons, ${ }^{6-10}$ since alternative viewpoints engender still deeper problems. Nonetheless, in many respects the mathematics is the least problematic issue; the physics it represents is often much less easy to grasp. ${ }^{11}$

Quandaries of the latter kind are also evident from the literature on complex light, in which a wide variety of pictorial representations can readily be found. ${ }^{12-14}$ In this respect, of course, there is no sense in attempting a visually meaningful depiction of the photon itself, and even to graphically represent the associated electromagnetic fields is a challenge - since in quantum optics those fields are operators, rather than simple vectors. Therefore it is logical that it is the beam structures that are most commonly depicted: there is some utility in such representations although, even then, no such depiction can adequately convey the underlying quantum physics. But it is notable that, individually, such illustrations will often reveal or support simple intuitive suppositions - and these in turn may sustain inferences or subliminal prejudices that can lead to an accidentally incorrect interpretation of the true physics, at the beam or photon level. This analysis aims to expose and underscore some of the issues that appear to merit much closer attention. 


\section{CONCEPTUAL ISSUES}

In its simplest consideration, the notion that photons in a beam of light might have a capacity to convey properties of the beam structure could be considered straightforward, signifying no more than the discrete quantization of energy levels for a corresponding radiation mode. Any complete set of modes should afford an acceptable basis for the radiation field, just as the plane waves that are more routinely deployed. Indeed, detailed calculations show that the quantum features of Laguerre-Gaussian (LG) modes, for example, can be developed in an exactly similar way. ${ }^{15}$ Nonetheless the mathematical premise for such a view quite evidently fails to satisfy physical intuition, for it is at least curious to consider that each individual photon might convey precisely representative values for properties of an entire whole beam - such as its radial structure, for example. The implications of such a view seem to surface more as a conceptual problem for vortex modes than, say, Hermite Gaussian or TEM modes - perhaps because vortices convey the more obviously 'tangible' property of orbital angular momentum. It is therefore not surprising, in consequence of such problems with structured light, that the distinct impression from casual users of the photon concept has often seemed to be that phenomena such as interference, or for example experiments involving sorting by topological charge, directly exhibit beam structure through classical forms of interaction - the notion that the same principles would operate at the photon level, remaining at best a logical inference.

However, experiments unequivocally show that individual photons can and do convey information on beam structure ${ }^{6-10}-$ a feature that is of course of overriding importance in the pursuit of optical communication and quantum information and applications. ${ }^{16-24}$ Although any direct measurement of an individual photon can only collapse a corresponding radiation state, experimental evidence clearly suggests that each photon itself exists, or exerts an influence at any instant in time it may engage with matter, over an extended region of space. It is a mistake to think that the pathway of any individual photon can properly be represented by a single infinitely thin line, as in ray optics. One of the clear results from Young's slits experiment is that when individual photons arrive at the screen they exhibit the effects of a superposition of trajectories from positions that are spatially separated by a distance large compared to the wavelength. In the same connection it is worth observing that those positions are also displaced in time from any point on the screen by different amounts. This also explains how an individual photon in a Laguerre Gaussian mode, or any other structured mode, can convey information from a source such as a spatial light modulator, whose programmed phase information spans pixels across distances much larger than the wavelength. It is therefore not unreasonable to consider that even a single photon in some sense 'occupies' the whole propagating transverse area of the beam. How else can a photon captured in an outer region still carry information connected with orbital angular momentum about the beam axis?

\section{PROPAGATION PHYSICS AND ITS REPRESENTATION}

The above considerations should inform choice amongst the various forms of diagrammatic construction intended to visually convey the nature of structured light. It is worth being aware of the background dominance - not exclusively carried over from introductory ray optics - of the concept of an infinitesimally thin route for each photon, in any beam of light in free space - which intuitively couples with the dictum that light travels in (or defines) a straight line. Even though we might not actively defend the detail of such a description, its essentially one-dimensional notion may subliminally permeate visualizations. The erroneous aspects of its foundation represent a falsehood that has not yet been fully dissipated, even by the enhanced focus on structural beam singularities. In fact, the problem here has a much broader basis, for even the plane waves that are most often used as the basis for describing quantized radiation in free space are well known to be unphysical. Defined in terms of an infinitely repeating set of planar wave-fronts, there is no uniquely definable orthogonal axis to ascribe to a photon propagation vector. More specifically we can say that the Poynting vector, with which the propagation is directly connected, is not anchored in the physical 3D space of linear dimensions - although, as an expression of linear momentum density, it can indeed be defined at any given time, at any point or region within that space. In short, momentum cannot be graphically represented by a vector portrayed in physical space - only in reciprocal (momentum) space.

Even before addressing the particular difficulties associated with figurative representations of structured light - and in particular any specifically quantum features - it is instructive to first consider a more familiar feature of optical radiation: 
the depiction of polarization state. Commonly, this is presented as a representation of the electric field in a classical limit, exhibiting a variation of the field with propagation distance $z$ at a given instant in time, as for example shown in Fig. 1 . Useful as such depictions may be, they ought not to be conceived as showing any kind of physical displacement from the propagation axis. Consider, for example, a plane-polarized electric field of amplitude $\mathrm{E}_{0}$, classically expressible as $\boldsymbol{E}(z)=$ $\mathrm{E}_{0} \hat{\boldsymbol{\imath}} \cos k z$, with $k$ the magnitude of the wave-vector, and unit vector $\hat{\boldsymbol{\imath}}$ denoting a field in the $x$-direction. It is worth emphasizing that this formula does not signify any physical displacement in the $x$-direction; the directly corresponding diagrammatic representation is a plot of the electric field magnitude $\mathrm{E}_{0} \cos k z$, not a distance in $z$ : the ordinate units in SI are $\mathrm{V} \mathrm{m}^{-1}$, not $\mathrm{m}$. Any inference that there is indeed a physical oscillation in the $x$-direction, weaving back and forth across the $z$-axis, can lead to unphysical speculations. ${ }^{25}$

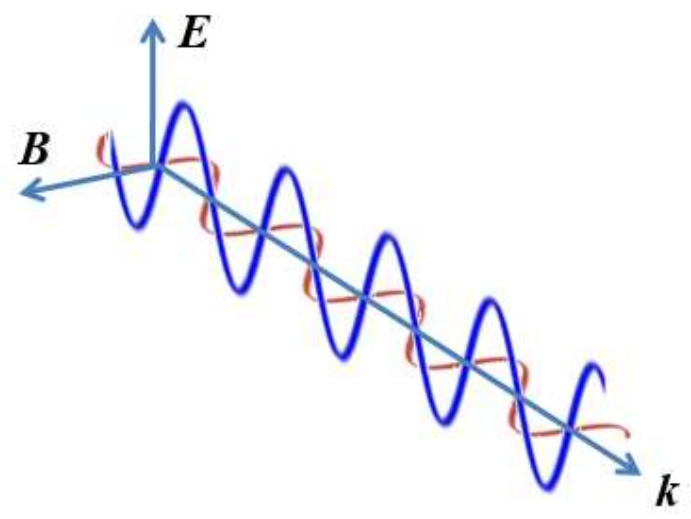

Figure 1. Conventional classical representation of the mutually orthogonal electric and magnetic field vectors, $\boldsymbol{E}$ and $\boldsymbol{B}$ respectively, and wave-vector $\boldsymbol{k}$.

Now in the depiction of structured light, a more elaborate variety of pictorial devices is employed. Two of the most important - each of them potentially misleading in detailed implementation - are representations of progressing wavefront and phase. There is indeed a connection; a wavefront is simply a surface of constant phase. Depictions of wavefront structure overcome many of the difficulties of pictorially representing local Poynting vectors; the latter may correctly be interpreted as being directed along local normals to the wave-front, even though their magnitudes are incommensurate with spatial representation. The local values of the Poynting vector (energy current density) are given by $\boldsymbol{E}(\boldsymbol{r}) \times \boldsymbol{B}(\boldsymbol{r}) \varepsilon_{0}$, where the local electric and magnetic fields are $\boldsymbol{E}(\boldsymbol{r})$ and $\boldsymbol{B}(\boldsymbol{r})$, respectively, at a position $\boldsymbol{r} .{ }^{26}$ In this connection, an optical vortex may be considered as light with a helically formed wavefront. Visually compelling as they may appear, the multi-colored helical surfaces often used to represent optical vortices are mostly misleading - especially where phase is depicted with color-coding. The wavefront is manifest as a moving surface in real three-dimensional space. When both polarization state and wavefront structure are shown together, their entirely different physical senses therefore need to be kept in mind.

Since an optical wavefront has same instantaneous phase everywhere, then for twisted light - just as for any other, simpler form - there is one wavelength in distance between successive intercepts on any parallel along the $z$ direction. This means that the wavefront surface for an optical vortex with a phase factor $\mathrm{e}^{\mathrm{i} m \phi}$ comprises $m$ interwined helical surfaces, each separated in distance from its nearest neighbor by one wavelength - see Fig. 2. At a distance $r$ from the axis, the pitch angle $\theta$ of the helicoidal surface normal is correspondingly set as shown in Fig. 3. While some reports cast the pitch angle as $\theta=m / k r,{ }^{27,28}$, this cannot be correct since it implies $\theta>\pi / 2$ for large enough $m$ - which would signify a reversal of handedness. The correct form of the relationship is $\tan \theta=m / k r$, correctly leading to $\theta=0$ for $m=0$ (and $\theta \rightarrow \pi / 2$ for $m \rightarrow \infty){ }^{29}$ Moreover, the usual form of expression is consistent with the small angle limit of the tangent. Of course the singular nature of the beam means there is zero intensity along the axis where $r=0$. (Note that both forms of result for the pitch angle dictate that the Poynting vector has an increasing transverse, 'inwardly' directed, component with increasing distance from the beam axis - signifying a transverse curvature that is seldom correctly represented in figurative illustrations. More often, a constant inward component is wrongly indicated.) Thus, for positions close to the beam axis, 
an uncertainty in measurability of the angular distinction between locally oriented wave-vectors, is asymptotically proportional to $m^{-1}$. Since the orbital angular momentum per photons is $m \hbar$, this deduction is entirely consistent with the angle-angular momentum uncertainty principle for structured light. ${ }^{30}$

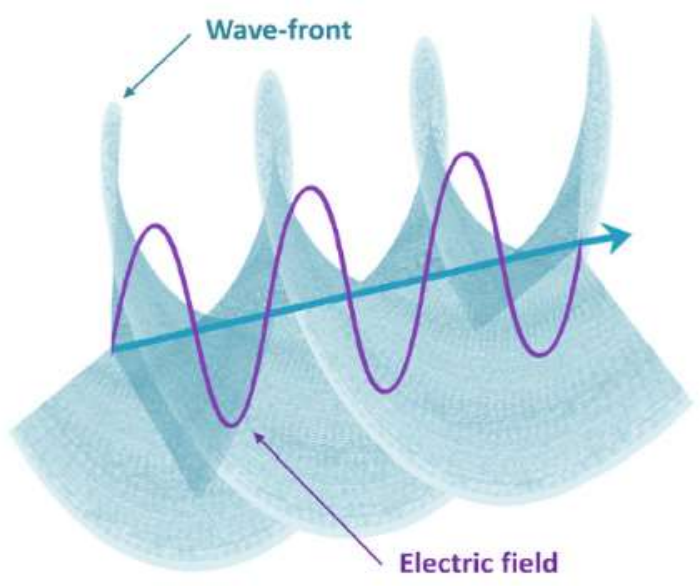

Figure 2. Propagation of a plane polarized optical vortex. Here, the left-handed helical wave-front has a topological charge $m=3$ signifying a wave-front comprising three intertwined helical surfaces, each component completing one full rotation about the axis over a span of three wavelengths. Adapted from ref. ${ }^{31}$

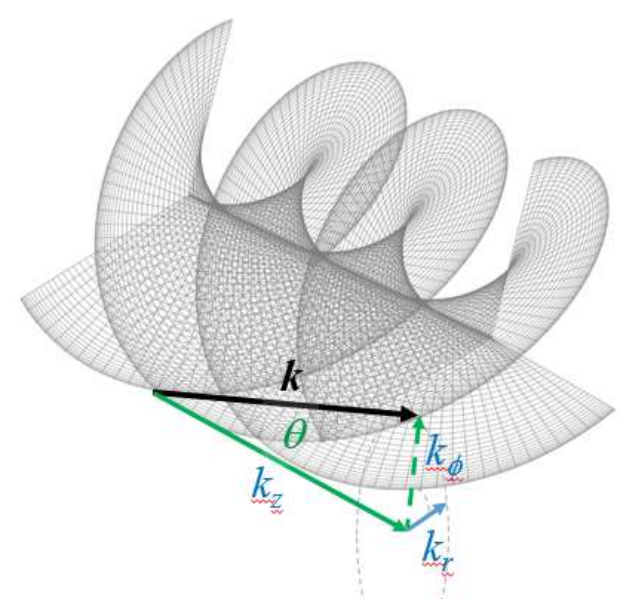

Figure 3. Axial, radial and azimuthal components of the local Poynting vector, $k_{z}, k_{r}$ and $k_{\phi}$ respectively, and the pitch angle $\theta$.

Depictions of optical phase ought in principle to offer better scope for meaningful depiction, since phase is a scalar quantity. Equally, however, there are still numerous pitfalls to avoid. As in all such depictions, it has first to be assumed that what is represented by any $3 \mathrm{D}$ visualization illustrates the phase at a specific point in time - with a reminder that greyscale representations cannot properly exhibit any continuity of phase. (Greyscale representations, shaded in density across the interval $(0,2 \pi)$, manifest meaningless discontinuities where an azimuthal angle approaches $2 \pi$ and the cycle starts over: it is possible for color representations to overcome this problem with a color-coded progression running through a circular spectrum - see Fig. 4(a) from ref. ${ }^{32}$ ). Optical phase is also, of course, only meaningful when registered against a particular reference - the location of a source, for example, or the phase of another optical field, present in the same 
locality. The issues of wavefront structure and phase variation across a beam become most intricate in the case of light with azimuthal phase variation, such as LG and Bessel beams. ${ }^{3}$ The rotation of phase around the propagation axis results in a phase singularity there, with vanishing electric and magnetic fields. Despite the impossibility of adequate graphical rendering, it is here worth recalling that vanishing fields and phase singularities are necessarily tempered by fundamental principles of quantum uncertainty. The electromagnetic fields are never entirely zero, and even for populated radiation modes there is always an uncertainty in phase connected with photon number. ${ }^{33-37}$ In this respect, the overlay of a greyor white-scale mapping of intensity might better reflect the physically meaningful, measurable attributes of the optical fields, as in the example shown in Fig. 4(b). ${ }^{32}$ One can go one stage further, depicting as a 3D density plot both phase and intensity, around and along the beam, as in Fig. 4(c). Mathematically coding such a representation is complicated by the partial occlusion of one side of the beam by the other.

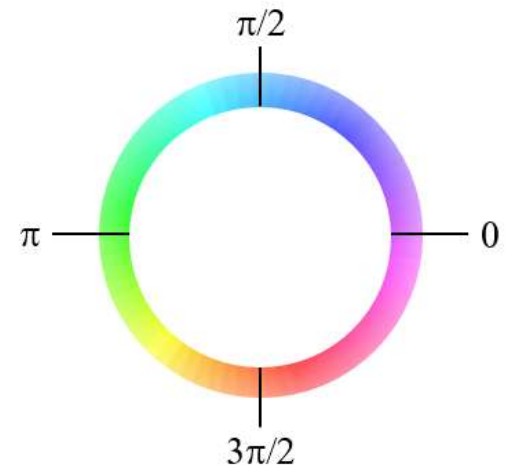

(a)

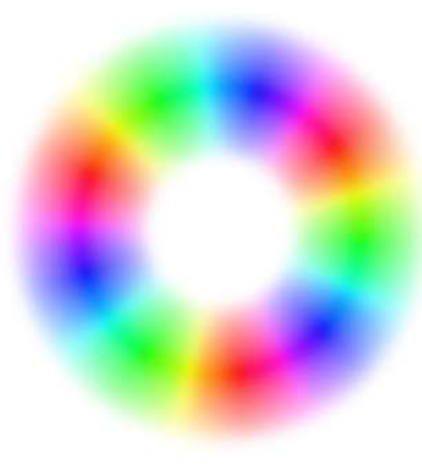

(b)

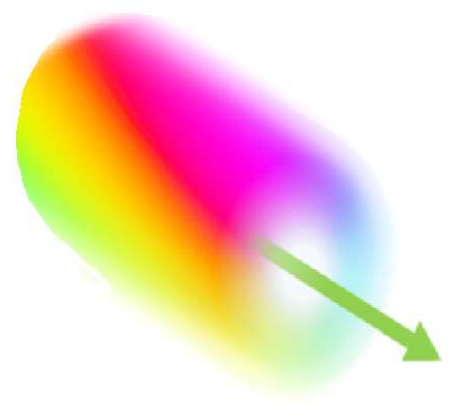

(c)

Figure 4. (a) Color-coding of azimuthal optical phase; (b) Transverse phase, intensity weighted by radial distribution of intensity for $\mathrm{LG}_{3,0}(m=3)$ beam; (c) $\mathrm{LG}_{1,0}(m=1)$ beam.

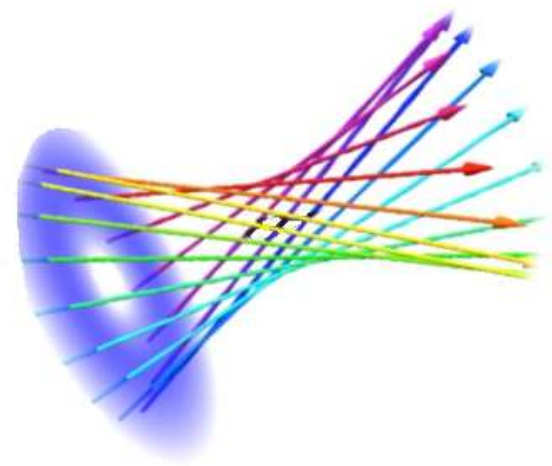

Figure 5. Vector bundle depiction of a vortex beam $(m=1)$. [Adapted from an original by S. Franke-Arnold]

It is easy to see the difficulties all this creates when one encounters depictions, whether textual or pictorial, of mode structures such as Airy, Bessel, Laguerre-Gaussian or even 'perfect' vortex modes. As observed earlier, the Poynting vector has a local direction at every point in space, though it is generally misleading to depict it as an arrow with linear dimensions, on a representation of physical, laboratory-related space. Considering local direction alone, as in Fig. 5, still produces an impression that the photons in any twisted beam are constantly heading out and away from the beam center. While this should not be taken as suggesting that there has to be a unique focal point in such a beam, the alternative appears 
to be a necessity for photons to constantly disappear from the beam and be replaced by others heading in for a glancing fly-by. If radiation modes with different wave-vector are orthogonal in Fock space, in what sense could a photon in one direction essentially turn into one travelling in a different direction? Posing the issue more carefully: if one were to think of the Poynting vectors as straight lines of photon propagation, it would have to be concluded that photons keep coming into and going out of existence all along the way - even in free space, in the absence of physical scattering.

Intriguingly, there is a flaw in the usually received commutation relation for the boson operators of distinct radiation modes, i.e. $\left[a_{\mathbf{k}}, a_{\mathbf{k}^{\dagger}}^{\dagger}\right]=\delta_{\mathrm{kk}^{\prime}}$. This expression cast in terms of a Kronecker delta is in fact improper, as it contains a pair of indices that do not refer to an orthogonal basis. Instead it should be written as a Dirac delta, $\left[a_{\mathbf{k}}, a_{\mathbf{k}^{+}}^{+}\right]=\left(8 \pi^{3} V\right)^{-1} \delta^{3}\left(\mathbf{k}-\mathbf{k}^{\prime}\right){ }^{29,38}$ Accordingly, the physical implication is that for photon propagation over a distance significantly smaller than the optical wavelength $\lambda$ (i.e. $V \ll \lambda^{3}$ ), measurably straight line propagation is not ensured. The position-momentum uncertainty comes strongly into play: over near-zone distances the photon essentially exhibits the character of a virtual particle. Any notional interplay of population between modes with marginally different wave-vector might then even then be rephrased as a question on the possibility of a superposition of offset mode states, with time-varying coefficients. Although such a representation would clearly be cast in an ill-fitting basis, that might not itself represent a fundamental objection, nor even the necessity to account for a discrete occupancy of each such mode: what is an insurmountable obstacle is the lack of any quantum interaction to modify such mode occupancy by the creation and annihilation of corresponding photons. We can therefore unambiguously dispense with the notion of any optical vortex as a continuously reforming vector bundle of plane waves.

\section{MATTER MATTERS}

Needless to say, in the absence of matter there is no mechanism for constant losses and gains between modes with different wave-vectors. Wherever photon creation and annihilation operators are involved - as for example in the application of any construct related to, or expressible in terms of, their commutation relation, interaction with matter has to be involved. So it becomes evident that the multimode picture, suggestive of off-track modal superposition is very misleading; LG photons are only created or annihilated through interaction with matter, when the twisted light is generated or detected. So is there any genuine physical basis for a commonly used, naïve description of photons in a vortex beam weaving around the axis? Does this have any bearing on the vaunted possibility of departures from the usual speed of light? ${ }^{39-41}$ It is not yet clear whether the correction described above might have any mathematical or interpretive bearing on the issue.

Ultimately, the only kinds of representation available to us - whether in theory or in visualizations - are those in which the 3D fabric of our recognizable world engages with light through material interaction. All the key issues for quantum phenomena have to be assessed through the lens of measurement. The meaningful registration of any photon requires identification, which signifies annihilation through local material interaction - generally in an atomic location far smaller than the wavelength. Generally, such detection produces a signal as a result of photon absorption that collapses the state: we cannot then know or even confidently deduce behavior prior to that event. Often, a cumulative electronic response emerges from the confluence of signals produced by individual photon absorption events. At the quantum level, the process through which each photon is absorbed is represented by projecting the initial state of the system, material plus incoming light, on to a final state in which the detector is in an excited state and the photon is spent. These system states are coupled by an interaction operator. Crucially, even in the case of resonant response to a specific optical frequency, the state projection can give a non-zero amplitude for a variety of optical states: in other words the measurement cannot be modespecific. The same is true, of course, for any input; no quantum detector can unequivocally resolve between modes of the same frequency, other than by polarization or beam structure.

To tackle this problem, one strategy may be to deploy antenna arrays supporting the direct, mode-specific input/output coupling with optical vortex radiation. ${ }^{42-44}$ Even such a delocalized array can do no more than respond to a corresponding projection of the optical transverse mode structure; it cannot be unambiguously mode-specific in its response. Considering photon absorption events in the electric-dipole approximation, then since the system response lacks any involvement of the optical wave-vector, the process will fail to clearly differentiate between differently structured modes, other than 
statistically in terms of relative photon flux in different transverse regions of the beam. The same applies to results calculated in the minimal coupling representation, whose results have been shown to be identical to the electric dipole results, once energy conservation is taken into account. ${ }^{45}$ It is only by going to higher order electric or magnetic multipolar transitions, beyond the electric or even magnetic dipole, that any sensitivity to the wave-vector comes into play: this is indeed a topic of current research activity. ${ }^{46-49}$

\section{REFERENCES}

[1] Andrews, D. L., [Structured Light and its Applications: An Introduction to Phase-Structured Beams and Nanoscale Optical Forces], Academic, Amsterdam, Boston (2008).

[2] Torres, J. P. and Torner, L., [Twisted Photons: Applications of Light with Orbital Angular Momentum], WileyVCH, Weinheim, Germany (2011).

[3] Andrews, D. L. and Babiker, M., [The Angular Momentum of Light], Cambridge University Press, Cambridge, UK (2013).

[4] Gbur, G., [Singular Optics], Wiley Online Library, (2016).

[5] Allen, L., Beijersbergen, M. W., Spreeuw, R. J. C. and Woerdman, J. P., "Orbital angular momentum of light and the transformation of Laguerre-Gaussian laser modes," Phys. Rev. A 45, 8185-8189 (1992).

[6] Galvez, E. J., Coyle, L. E., Johnson, E. and Reschovsky, B. J., "Interferometric measurement of the helical mode of a single photon," New J. Phys. 13, 053017 (2011).

[7] Cheng, X. and Galvez, E. J., "Imaging single photons in non-separable states of polarization and spatial-mode," Proc. SPIE 9225, 922506 (2014).

[8] Tempone-Wiltshire, S. J., Johnstone, S. P. and Helmerson, K., “Optical vortex knots-one photon at a time,” Sci. Rep. 6, 24463 (2016).

[9] Ndagano, B., Nape, I., Perez-Garcia, B., Scholes, S., Hernandez-Aranda, R. I., Konrad, T., Lavery, M. P. and Forbes, A., "A deterministic detector for vector vortex states," Sci. Rep. 7, 13882 (2017).

[10] Wang, X.-L., Cai, X.-D., Su, Z.-E., Chen, M.-C., Wu, D., Li, L., Liu, N.-L., Lu, C.-Y. and Pan, J.-W., "Quantum teleportation of multiple degrees of freedom of a single photon," Nature 518, 516 (2015).

[11] Galvez, E. J. and Zhelev, N., "Orbital angular momentum of light in optics instruction," in [Education and Training in Optics and Photonics](Optical Society of America, 2007), p. ESB3.

[12] Allen, L., Padgett, M. J. and Babiker, M., “The orbital angular momentum of light,” Prog. Opt. 39, 291-372 (1999).

[13] Allen, L., Barnett, S. M. and Padgett, M. J., [Optical Angular Momentum], Taylor \& Francis Group, Bristol (2003).

[14] Rubinsztein-Dunlop, H., Forbes, A., Berry, M. V., Dennis, M. R., Andrews, D. L., Mansuripur, M., Denz, C., Alpmann, C., Banzer, P., Bauer, T., Karimi, E., Marrucci, L., Padgett, M., Ritsch-Marte, M., Litchinitser, N. M., Bigelow, N. P., Rosales-Guzmán, C., Belmonte, A., Torres, J. P., Tyler, W. N., Baker, M., Gordon, R., Stilgoe, A. B., Romero, J., White, A. G., Fickler, R., Willner, A. E., Xie, G., McMorran, B. and Weiner, A. M., "Roadmap on structured light," J. Opt. 19, 013001 (2017).

[15] Dávila Romero, L. C., Andrews, D. L. and Babiker, M., “A quantum electrodynamics framework for the nonlinear optics of twisted beams," J. Opt. B: Quantum Semiclass. Opt. 4, S66-S72 (2002).

[16] Mair, A., Vaziri, A., Weihs, G. and Zeilinger, A., "Entanglement of the orbital angular momentum states of photons," Nature 412, 313-316 (2001).

[17] Lavery, M. P., Fraine, A., Roberston, D., Sergienko, A., Courtial, J., Wilner, A. E. and Padgett, M. J., "The measurement and generation of orbital angular momentum using an optical geometric transformation," Proc. SPIE 8610, 86100J (2013).

[18] Malik, M., Mirhosseini, M., Lavery, M. P., Leach, J., Padgett, M. J. and Boyd, R. W., "Direct measurement of a 27-dimensional orbital-angular-momentum state vector," Nat. Commun. 5, 3115 (2014).

[19] Mirhosseini, M., Magaña-Loaiza, O. S., O’Sullivan, M. N., Rodenburg, B., Malik, M., Lavery, M. P. J., Padgett, M. J., Gauthier, D. J. and Boyd, R. W., "High-dimensional quantum cryptography with twisted light," New J. Phys. 17, 033033 (2015).

[20] Potoček, V., Miatto, F. M., Mirhosseini, M., Magaña-Loaiza, O. S., Liapis, A. C., Oi, D. K. L., Boyd, R. W. and Jeffers, J., “Quantum Hilbert Hotel,” Phys. Rev. Lett. 115, 160505 (2015). 
[21] Jurado-Navas, A., Tatarczak, A., Lu, X., Olmos, J. J. V., Garrido-Balsells, J. M. and Monroy, I. T., "850-nm hybrid fiber/free-space optical communications using orbital angular momentum modes," Opt. Express 23, 33721-33732 (2015).

[22] Chen, M., Dholakia, K. and Mazilu, M., "Is there an optimal basis to maximise optical information transfer?," Sci. Rep. 6, 22821 (2016).

[23] Hiesmayr, B. C., de Dood, M. J. A. and Löffler, W., "Observation of four-photon orbital angular momentum entanglement," Phys. Rev. Lett. 116, 073601 (2016).

[24] Coles, M. M., "An upper bound on the rate of information transfer in optical vortex beams," Laser Physics Letters 15, 095202 (2018).

[25] Li, H., "Evanescent wave of a single photon," Opt. Eng. 52, 074103 (2013).

[26] Zangwill, A., [Modern Electrodynamics], Cambridge University Press, Cambridge (2013).

[27] Yeganeh, M., Rasouli, S., Dashti, M., Slussarenko, S., Santamato, E. and Karimi, E., "Reconstructing the Poynting vector skew angle and wavefront of optical vortex beams via two-channel moiré deflectometery," Opt. Lett. 38, 887-889 (2013)

[28] Bekshaev, A. Y., Kurka, I., Mohammed, K. and Slobodeniuk, I., "Wide-slit diffraction and wavefront diagnostics of optical-vortex beams," Ukr. J. Phys. Opt. 16, 17-23 (2015).

[29] Andrews, D. L. and Forbes, K. A., "Quantum features in the orthogonality of optical modes for structured and plane-wave light," Opt. Lett. 43, 3249-3252 (2018).

[30] Franke-Arnold, S., Barnett, S. M., Yao, E., Leach, J., Courtial, J. and Padgett, M., "Uncertainty principle for angular position and angular momentum," New J. Phys. 6, 103 (2004).

[31] Andrews, D. L., "Photon-based and classical descriptions in nanophotonics: a review," J. Nanophoton. 8, 081599-081599 (2014).

[32] Andrews, D. L. and Bradshaw, D. S., [Introduction to Photon Science and Technology], SPIE Press, Bellingham (2018).

[33] Power, E. A., [Introductory Quantum Electrodynamics], American Elsevier Pub. Co., New York (1965).

[34] Louisell, W. H., [Quantum Statistical Properties of Radiation], Wiley, New York (1973).

[35] Milonni, P. W., [The Quantum Vacuum: An Introduction to Quantum Electrodynamics], Academic Press, San Diego (1993).

[36] Loudon, R., [The Quantum Theory of Light], Oxford University Press, Oxford (2000).

[37] Barnett, S. M. and Vaccaro, J. A., [The Quantum Phase Operator: A Review], Taylor \& Francis, (2007).

[38] Williams, M. D., Bradshaw, D. S. and Andrews, D. L., "Quantum issues with structured light," Proc. SPIE 9764, 976407 (2016).

[39] Bouchard, F., Harris, J., Mand, H., Boyd, R. W. and Karimi, E., "Observation of subluminal twisted light in vacuum," Optica 3, 351-354 (2016).

[40] Bareza, N. D. and Hermosa, N. J. S. r., "Subluminal group velocity and dispersion of Laguerre Gauss beams in free space," Sci. Rep. 6, 26842 (2016).

[41] Lyons, A., Roger, T., Westerberg, N., Vezzoli, S., Maitland, C., Leach, J., Padgett, M. J. and Faccio, D., "How fast is a twisted photon?," Optica 5, 682-686 (2018).

[42] Williams, M. D., Coles, M. M., Saadi, K., Bradshaw, D. S. and Andrews, D. L., "Optical vortex generation from molecular chromophore arrays," Phys. Rev. Lett. 111, 153603 (2013).

[43] Coles, M. M., Williams, M. D., Saadi, K., Bradshaw, D. S. and Andrews, D. L., "Chiral nanoemitter array: A launchpad for optical vortices," Laser \& Photon. Rev. 7, 1088-1092 (2013).

[44] Zang, X. and Lusk, M. T., "Twisted molecular excitons as mediators for changing the angular momentum of light,” Phys. Rev. A 96, 013819 (2017).

[45] Power, E. A. and Thirunamachandran, T., "On the nature of Hamiltonian for interaction of radiation with atoms and molecules: (e/mc)p.A, - $\mu$.E, and all that," Am. J. Phys. 46, 370-378 (1978).

[46] Forbes, K. A. and Andrews, D. L., "Optical orbital angular momentum: twisted light and chirality,” Opt. Lett. 43, 435-438 (2018).

[47] Forbes, K. A., "Raman Optical Activity Using Twisted Photons," Phys. Rev. Lett. 122, 103201 (2019).

[48] Forbes, K. A. and Andrews, D. L., "Spin-orbit interactions and chiroptical effects engaging orbital angular momentum of twisted light in chiral and achiral media," Phys. Rev. A 99, 023837 (2019).

[49] Forbes, K. A. and Andrews, D. L., "Enhanced optical activity using the orbital angular momentum of structured light,” Phys. Rev. Res. 1, 033080 (2019). 